\title{
Current Smoking is a Risk Factor for the Irregular Surface and Calcification of Carotid Plaque in Men
}

\author{
Xiangli $X u^{\prime}$ \\ Fubo Zhou ${ }^{2}$ \\ Yang $\mathrm{Hua}^{2}$ \\ Beibei Liu ${ }^{2}$ \\ Lili Wang ${ }^{2}$ \\ Weihong $\mathrm{Hou}^{2}$ \\ Mingyu $\mathrm{Xia}^{2}$ \\ 'Department of Ultrasound, The Second \\ Hospital of Harbin, Harbin, People's \\ Republic of China; ${ }^{2}$ Department of \\ Vascular Ultrasonography, Xuanwu \\ Hospital, Capital Medical University, \\ Beijing, People's Republic of China
}

Correspondence: Yang Hua Department of Vascular Ultrasonography, Xuanwu Hospital, Capital Medical University, No. 45 Changchun Road, Beijing, 100053, People's Republic of China

Tel +86-10-83198923

Fax +86-10-83198967

Email dryanghua99@163.com
Objective: To explore whether current smoking could influence plaque characteristics and determine its correlation to the irregular surface and calcification of carotid plaque.

Methods: Three hundred and seventeen patients with severe carotid atherosclerosis stenosis (SCAS) detected by color duplex flow imaging (CDFI) and confirmed by CT angiography (CTA) were recruited. The results of laboratory parameters were collected by using electronic database of the hospital. Computerized tomography (CT) scanning and high-resolution ultrasonography were performed for assessment of plaque morphology, respectively.

Results: All enrolled smokers and non-smokers had no significant difference among all characteristics not related to smoking. CT scanning could efficiently identify the difference among enrolled smokers and non-smokers not only for the characteristics related to smoking but also the onsets of carotid plaque. Surface morphology was also efficiently detected by ultrasonography. Further ridge trace analysis showed that ultrasonography is efficient for diagnosis of calcified plaque compared with gold standard for plaque diagnosis. Further correlation analysis showed that ultrasonography parameters could offer reliable evidence for plaque scores, which was associated with age index. Ultrasonography parameters could efficiently differentiate plaque morphologies among enrolled smokers and never-smokers.

Conclusion: Current smoking was positively associated with plaque calcification onsets, and smoking cessation could efficiently attenuate such injury. High-frequency ultrasound can clearly distinguish the details of calcification with promising clinical significance for current smoking patients.

Keywords: smoking, ultrasonography, carotid plaque, calcification

\section{Introduction}

Plaque calcification is negatively related with the prognosis of atherosclerosis, which is associated with multiple adverse cardiovascular events. Plenty of clinical evidence has proven that carotid calcification accelerated and contributed to a higher risk of cardiovascular morbidity and mortality in various disease subpopulation, including coronary disease, hypertension, diabetes mellitus and chronic kidney disease. ${ }^{1}$ In addition, carotid plaque calcification is an independent predictor of carotid calcification and long-term adverse vascular events, and it has been widely proven that carotid plaque calcification are associated with plaque progression and vulnerability. Epidemiologic studies demonstrated that the prevalence of carotid plaque calcification or irregular surface in the general population is nearly $60 \%$ for women and $70 \%$ for men aged from 41 to 80 years. ${ }^{2,3}$ Moreover, in the high-risk sub-population, this rate increased dramatically and caused various adverse prognosis, for example, $81 \%$ of diabetes mellitus patients suffer from 
plaque calcification while almost all end-stage renal disease patients were diagnosed with carotid calcification., ${ }^{4,5}$

Considering irregular surface and calcification of carotid plaque are associated with long-term comorbidities and clinical prognosis, measurement for calcification of carotid plaque has been increasingly used to enable accurate cardiovascular risk assessments of these patients. It is originally described and reported that ultrasonography could be used for diagnosing bone-like artery wall morphologies, then resigned to a passive side-effect of aging, ${ }^{6} \mathrm{VC}$ has reemerged as a tightly regulated, complex disease, resembling that of bone mineralization. ${ }^{7}$ Moreover, there are 2 main types of carotid plaque: atherosclerosis-associated intimal or diabetes mellitus and CKD-associated medial calcification, while it has been reported that both types of plaque are associated with unhealthy lifestyles. ${ }^{8-10}$ In addition, researchers have demonstrated that some factors even directly influenced deposition of calcium phosphate hydroxyapatite crystals into the extracellular matrix. ${ }^{11,12}$

Smoking is a normal unhealthy lifestyle and risk factor, especially in male population. China remains both the world's largest producer and consumer of tobacco products with an estimated smoking population of 350 million people. ${ }^{13}$ Recent study has demonstrated that smoking is associated with vascular injury and atherosclerosis progression. $^{14,15}$ Furthermore, the rate of carotid plaque seemed to be higher in the smoking-habit population, but the efficient diagnosis method warranted more clinical evidence. On the other hand, it is unclear whether smoking cessation could attenuate calcification of carotid plaque.

The present study is aimed to explore the detailed diagnosis effect of combination between traditional CT and ultrasonography and determine the effect of current smoking and smoking cessation on calcification of carotid plaque.

\section{Methods}

\section{Patients Recruitment}

Three hundred and seventeen patients with severe carotid atherosclerosis stenosis (SCAS) detected by color duplex flow imaging (CDFI) and confirmed by $\mathrm{CT}$ angiography (CTA) were recruited in this study. Patients were divided into calcified plaque or non-calcified plaque according to CTA imaging of SCAS-responsible plaque and the characteristics of plaques evaluated by high frequency ultrasound.
Our clinical study recruited patients from clinical database, which is listed at www.clinicaltrials.gov (NCT02306616). This study is approved by the ethics committee of Xuanwu Hospital, Capital Medical University (No.2014018). Written informed consent was obtained.

The SCAS is defined for study inclusion according to the recommended diagnostic criteria of the SCAS diagnosis criteria issued by the Chinese Society of Cardiology. ${ }^{16}$ In detail, patients were enrolled in the study if they fulfilled the following criteria: (1) carotid atherosclerosis diagnosis by CTA, and (2) treatment with stent deployment.

The exclusion criteria are as follows: (1) patients who did not have stent deployment or had undergone surgical revascularization procedures or had undergone biodegradable stent implantation, (2) patients who had serum creatinine higher than $2.0 \mathrm{mg} / \mathrm{dL}$, (3) patients who had atherosclerosis lesion, (4) pump failure Killip I grade, (5) on dialysis or end stage kidney disease.

\section{Data Collection}

The results of laboratory parameters were collected using electronic database of the hospital. For parameters except for lipid profile and glucose levels, reports of admission blood samples were used. For lipid profile, the results of fasting blood samples collected within the 24-h of hospitalization were used. Total cholesterol, HDL-C and triglyceride levels were measured enzymatically (Architect c-Systems, Abbott, IL) and LDL-C levels were measured from these lipid parameters with Friedewald formula. Complete blood count (CBC) testing utilized clinical laboratory methods (Coulter LH 780 Hematology Analyzer, Beckman Coulter Ireland Inc., Mervue, Galway, Ireland) for hemoglobin.

\section{CT Scanning for Calcified Plaque}

Computerized tomography (CT) scanning was performed using a second-generation, dual-source 64-slice CT scanner (Somatom Sensation 64, Siemens, Forchheim, Germany). According to the CT plaque image, the subjects were divided into calcified plaque group and non-calcified plaque group.

\section{High-Resolution Ultrasonography for Assessment of Plaque Morphology}

Plaque characteristics were evaluated by high frequency ultrasound using cine and all the results were recorded. 
The plaque ultrasonography is obtained by the $3-9 \mathrm{MHz}$ linear array transducer (Philips EPIQ7, Bothell Everett Highway, Washington, USA) or a $4-8 \mathrm{MHz}$ micro-convex array probe (Hitachi VISION Preirus, HITACHI, Tokyo, Japan) and the plaque features include echogenicity, thickness, and surface morphology. All high-resolution ultrasonography examinations were performed by the same examiner, experienced in vascular ultrasound for 9 years. The examiner was blinded to clinical findings and CT results.

\section{Statistical Analysis}

All data were presented as a mean \pm SD or a median [25th and 75th percentile] for parametric variables and as percentages for categorical variables. Continuous variables were checked for the normal distribution assumption using Kolmogorov-Smirnov statistics. Ridge trace was performed for baseline characteristics of subjects classified according to smoking status. Differences between the groups were evaluated using Student's $t$-test or MannWhitney $U$-test as appropriate. Binary logistic regression analysis was performed and forward stepwise multivariable regression models were created to identify the independent predictors of CIN. Variables with $\mathrm{p}<0.10$ in univariable analysis were included in the multivariable model. Logistic regression analyses were used to investigate the univariable and multivariable predictors with $95 \%$ confidence intervals (CI). A p value $<0.05$ was considered statistically significant. All statistical studies were carried out using Statistical Package for Social Sciences software (SPSS 20.0 for Windows, SPSS Inc., Chicago, IL).

\section{Results}

\section{Comparison of Baseline Data Between Current Smokers and Former Smokers}

To establish a baseline database with comprehensive risk factors profile, we analyzed more than 20 factors for all recruited patients. Compared with former smokers, most factors in current smokers group had no significant difference ( $\mathrm{P}>0.05$, Table 1$)$, including sex, BMI, hypertension, hyperlipidemia, diabetes mellitus, family history of $\mathrm{CHD}$, chronic heart failure, chronic kidney failure, OMI, post PCI, post CABG, DBp, HR, and HbA1C. All these findings suggested that the two groups had a robust comparability at baseline for further analysis. On the other hand, age, triglyceride, smoking duration (years) and daily smoking amount were different in the current smokers compared with former smokers, which could be explained by the fact that current smokers had an obvious longer smoking history than former smokers.

\section{CT Scanning is Efficient for Diagnosis of Calcified Plaque}

As shown in Table 1, all enrolled smokers and non-smokers had no significant difference among all characteristics not related to smoking. In addition, characteristics related to smoking showed significant difference, including Smoking duration (years) and Daily smoking amount. Moreover, CT

Table I Baseline Characteristics of Subjects Classified According to Smoking Status

\begin{tabular}{|c|c|c|c|c|}
\hline Demographics & Never-Smoker $(n=79)$ & Current Smoker $(n=144)$ & Former Smoker $(n=94)$ & $P$-value \\
\hline Age (years) & $68 \pm 9$ & $64 \pm 8$ & $68 \pm 8$ & $<0.001$ \\
\hline BMI $\left(\mathrm{kg} / \mathrm{m}^{2}\right)$ & $25.0 \pm 2$ & $24.7 \pm 3.0$ & $25.2 \pm 2.9$ & 0.541 \\
\hline Systolic blood pressure $(\mathrm{mm} \mathrm{Hg})$ & $|33.1 \pm| 4.6$ & $133.3 \pm 13.2$ & $136.9 \pm 13.9$ & 0.092 \\
\hline Diastolic blood pressure $(\mathrm{mm} \mathrm{Hg})$ & $77.2 \pm 8.8$ & $76.8 \pm 8.5$ & $79.6 \pm 8.8$ & 0.054 \\
\hline Total cholesterol (mg/dL) & $3.6 \pm 1.1$ & $3.5 \pm 0.8$ & $3.4 \pm 0.8$ & 0.298 \\
\hline HDL-cholesterol (mg/dL) & $1.3 \pm 0.3$ & $1.2 \pm 0.4$ & $1.3 \pm 0.3$ & 0.974 \\
\hline LDL-cholesterol (mg/dL) & $2.1 \pm 0.9$ & $2.0 \pm 0.7$ & $2.1 \pm 0.7$ & 0.944 \\
\hline Triglyceride (mg/dL) & $1.9 \pm 1.0$ & $1.6 \pm 1.1$ & $1.4 \pm 0.6$ & 0.031 \\
\hline Glycemia (mmol/L) & $6.2 \pm 2.2$ & $6.5 \pm 2.5$ & $5.8 \pm 1.9$ & 0.069 \\
\hline Diabetes mellitus & $32 / 47$ & $57 / 87$ & $33 / 61$ & 0.718 \\
\hline Hypertension & $56 / 23$ & $99 / 45$ & $68 / 26$ & 0.833 \\
\hline Dyslipidemia & $30 / 49$ & $43 / 101$ & $33 / 61$ & 0.433 \\
\hline Smoking duration (years) & 0 & $38 \pm 9$ & $27 \pm 12$ & $<0.001$ \\
\hline Daily smoking amount & 0 & $20.6 \pm 11.1$ & $18.5 \pm 11.8$ & $<0.001$ \\
\hline
\end{tabular}


scanning could efficiently identify the difference among enrolled smokers and non-smokers not only for the characteristics related to smoking, but also the irregular surface and calcification of carotid plaque (Figure 1).
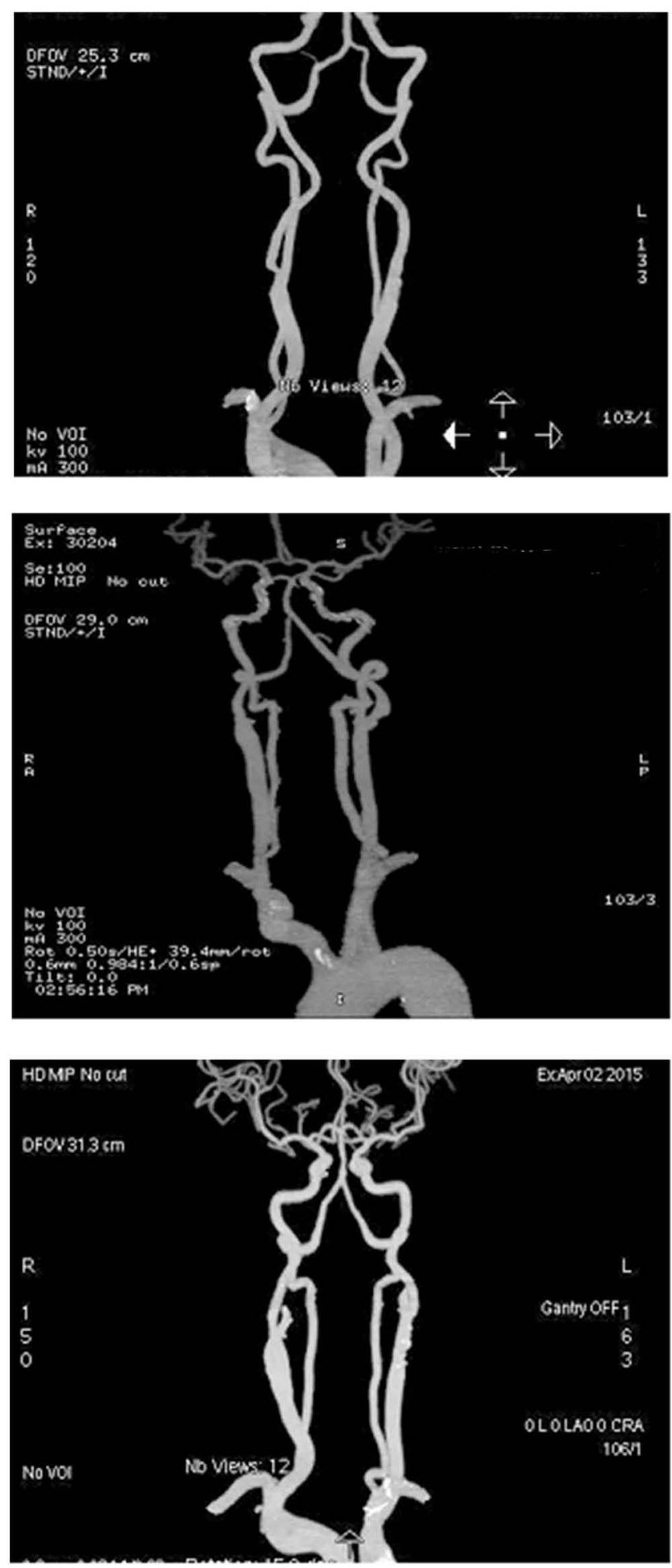

Ultrasonography Determined Calcified Plaque and Other Plaque Morphology

Ultrasonography was performed to determine calcified plaque and other plaque morphology. Analysis showed
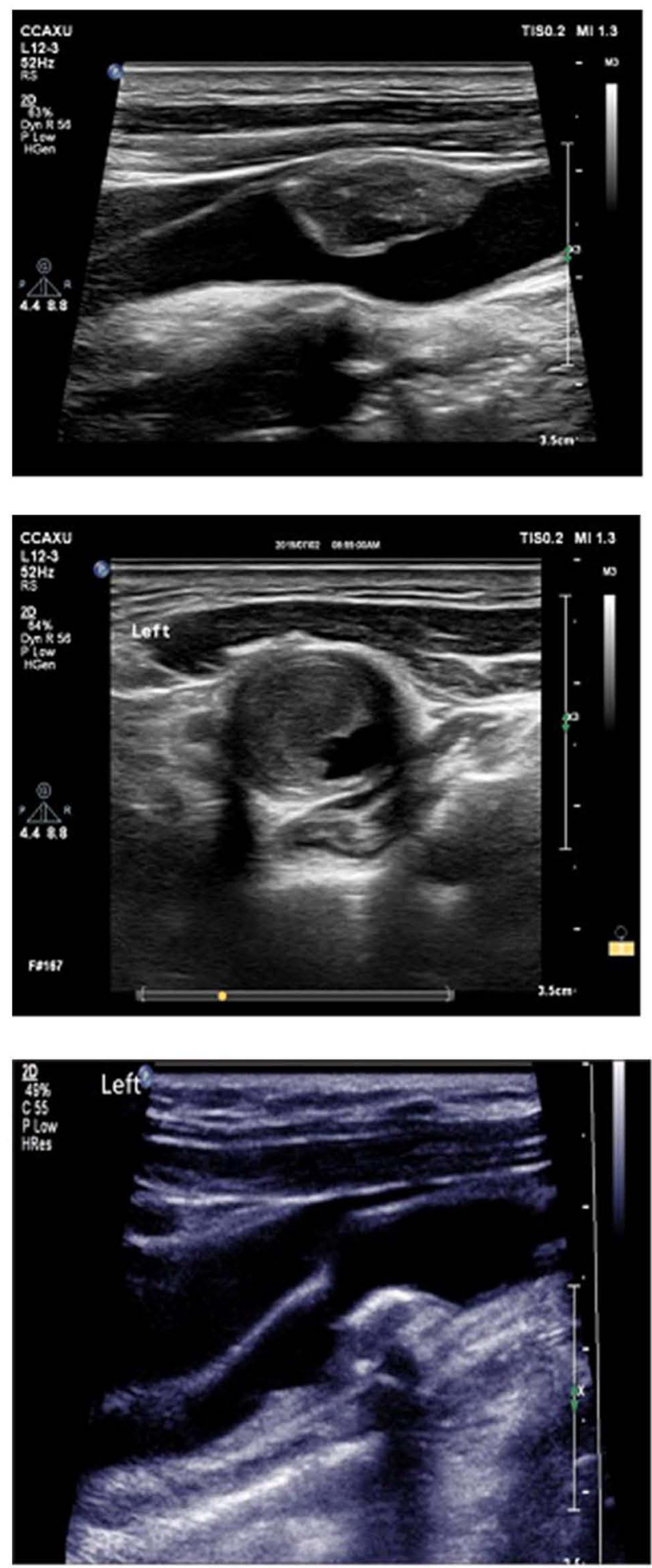

Figure I Image of irregular surface and calcification of carotid plaque for enrolled smokers and non-smokers, including CT scanning (left panel) and ultrasonography (right panel). 
that presence of calcification could be identified by ultrasonography. In addition, surface morphology was also efficiently detected by ultrasonography (Table 2). Further ridge trace analysis showed that ultrasonography is efficient for diagnosis of calcified plaque compared with gold standard for plaque diagnosis (Figure 2). Further correlation analysis showed that ultrasonography index could offer reliable evidence for plaque scores, which was associated with age index (Figure 3). Moreover, ultrasonography index could efficiently differentiate the calcified plaque and other plaque morphology among enrolled smokers and non-smokers (Figure 3).

\section{Multivariate Regression Analysis of Related Risk Factors Between Calcification and Plaque Surface Morphology}

After adjusting the risk factors, current smoking (OR 3.74 95\% 1.2-11.6) is still an independent risk factor for plaque calcification, while former smoking (OR $0.336,95 \% \mathrm{CI}$ $0.14-0.78$ ) is an independent prevention factor for plaque calcification, which also proves that smoking cessation can indeed reduce the formation of calcification. However, there was no significant correlation between former smoking and plaque surface irregularity. Age is also a risk factor for plaque surface irregularity (Table 3 ).

\section{Discussion}

Our study has some important strengths. First, we combined the advantages of both $\mathrm{CT}$ and ultrasound imaging methods to assess plaque calcification. $\mathrm{CT}$ is very sensitive and accurate for calcification and was used to determine calcified plaque. High-frequency ultrasound can clearly distinguish the details of calcification and was used to observe the location and type of calcification. Second, few articles have focused on calcification in the past. In the present study, we excluded possible confounding factors as much as possible and found that smoking is a very important risk factor for calcification, which was not mentioned in previous literature. Smoking is an artificially controllable risk factor, and the present study provides a theoretical basis for advocating smoking cessation.

CT scanning is the gold standard diagnosis modality of patients with plaque calcification, especially for ones with unhealthy lifestyle. ${ }^{17,18}$ Smoking has become an important predictor for plaque calcification. Prognostic importance of plaque calcification has been shown in patients with smoking habit, including current smoking and smoking history. ${ }^{19}$ In our study, we mainly focused on the current smoking patients with plaque calcification, which has been provn as the major group subject to further adverse prognosis. In the field of potential mechanisms, oxidative stress, inflammation and renal ischemia/reperfusion constitute the main pathophysiological mechanisms of plaque calcification, while current smoking was proved with more risk potential compared with smoking history patients, suggesting that smoking cessation could help to reduce risk of plaque calcification. ${ }^{20,21}$ On the other hand, impaired plaque calcification is a risk factor for cardiovascular diseases and in patients with STEMI, admission renal function or worsening during the hospitalization has been shown to indicate higher risk for adverse events. $^{22,23}$

Similar with imaging scan for plaque calcification in previous reports, in-hospital worsening condition of CT

Table 2 Carotid Plaque Characteristics According to Smoking Status

\begin{tabular}{|c|c|c|c|c|}
\hline Plaque Parameters & Never-Smoker $(n=79)$ & Current Smoker $(n=\mid 44)$ & Former Smoker $(n=94)$ & $P$-value \\
\hline Echogenicity & & & & 0.244 \\
\hline Homogeneous & 2 & 0 & 2 & \\
\hline Mainly high & 6 & 17 & 6 & \\
\hline Mainly medium & 50 & 88 & 52 & \\
\hline Mainly low & 21 & 39 & 34 & \\
\hline Thickness (mm) & $5.8 \pm 1.4$ & $5.7 \pm 1.3$ & $5.9 \pm 1.2$ & 0.833 \\
\hline Surface morphology & & & & 0.004 \\
\hline Regular & 26 & 21 & 26 & \\
\hline Irregular & 53 & 123 & 68 & \\
\hline Presence of calcification & 59 & 139 & 84 & $<0.001$ \\
\hline
\end{tabular}




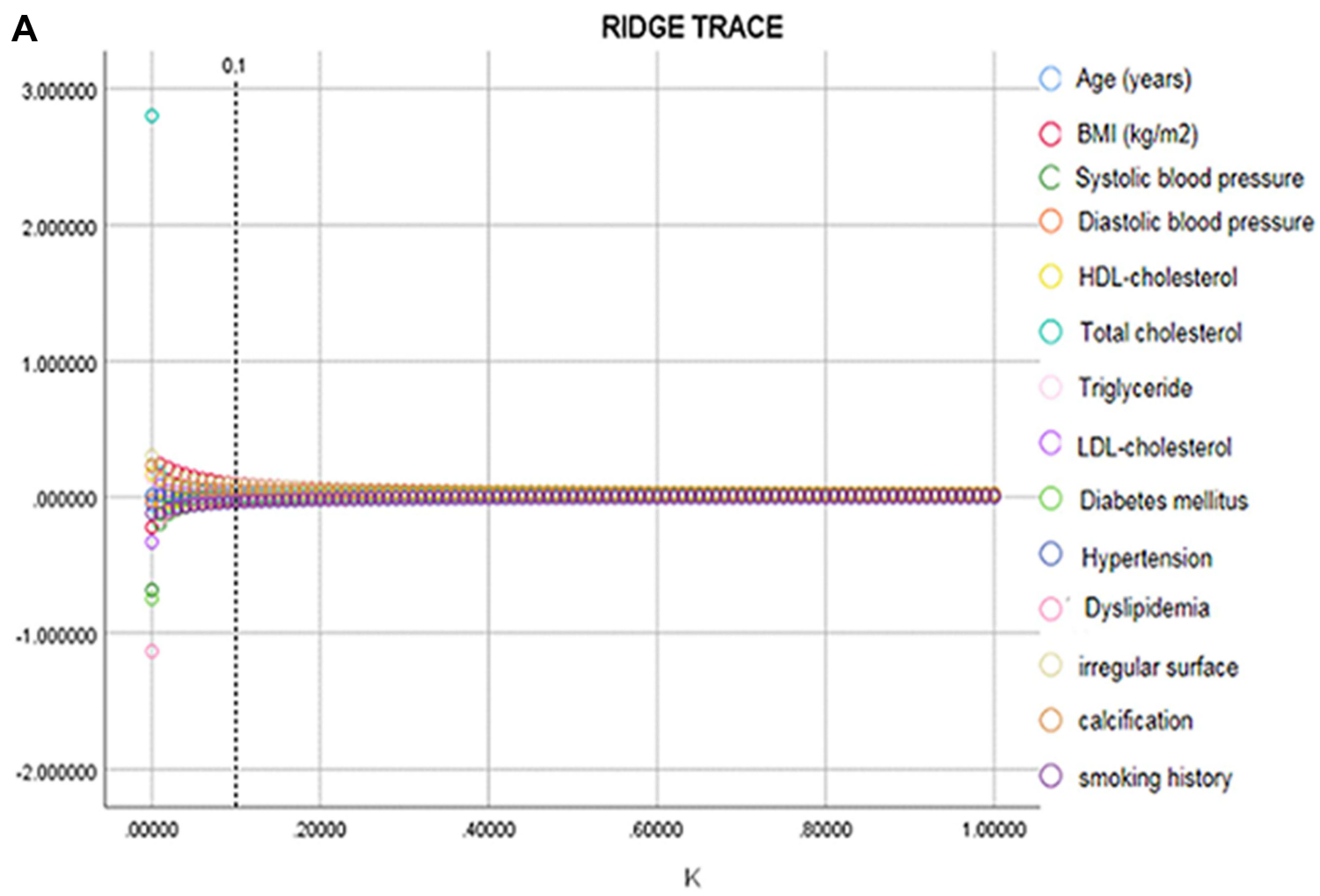

B

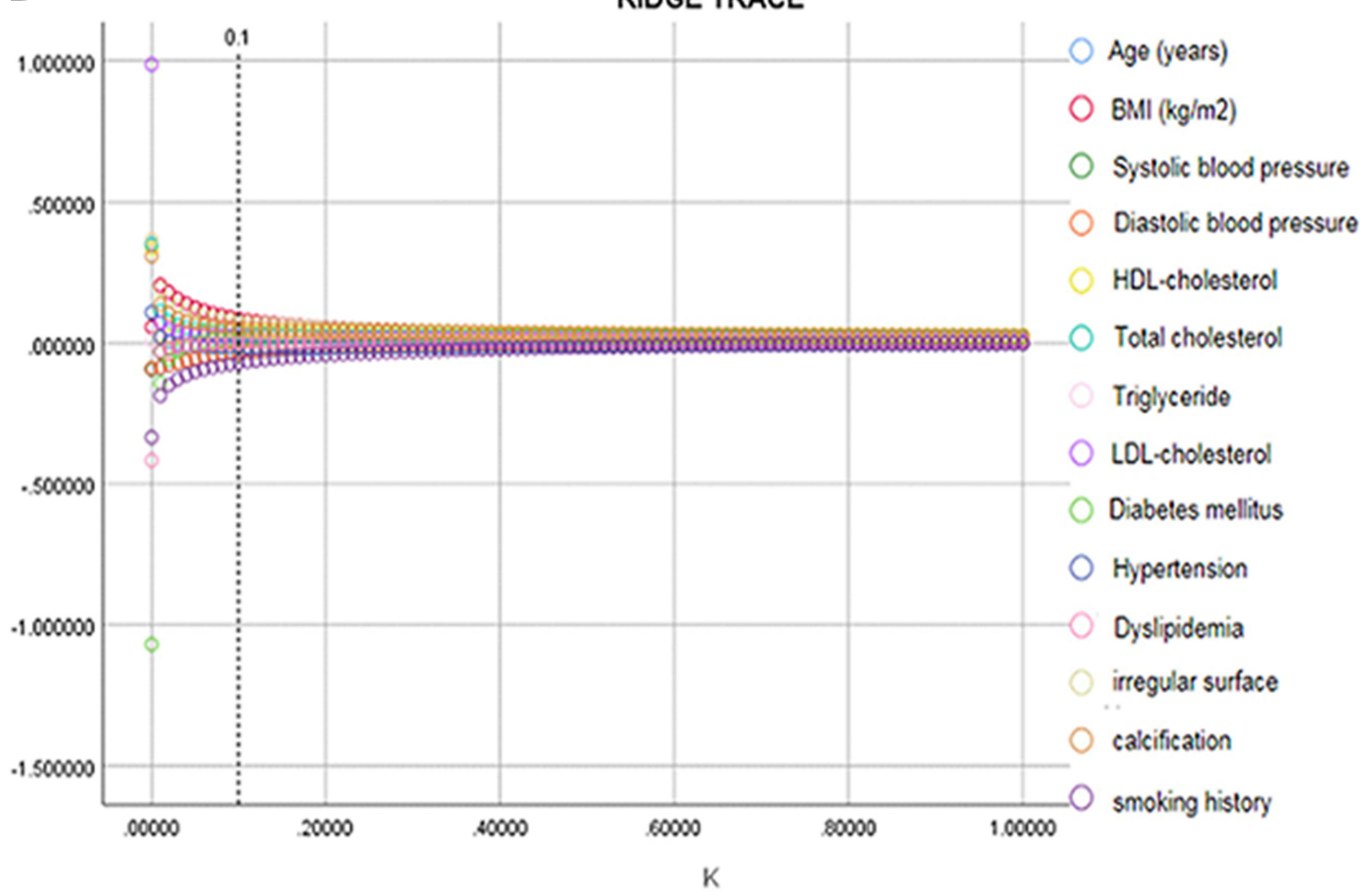

Figure 2 Ridge trace analysis for different examination strategies. (A) Gold standard for plaque diagnosis. (B) Ultrasonography for determination of calcified plaque.

result is also correlated with plaque calcification. history ones as control. Retrospective analysis demonAccordingly, we recruited sufficient patients with current strated that CT indeed had accurate diagnosis effect for smoking and prepared adequate amount of smoking plaque calcification, which is consistent with previous 


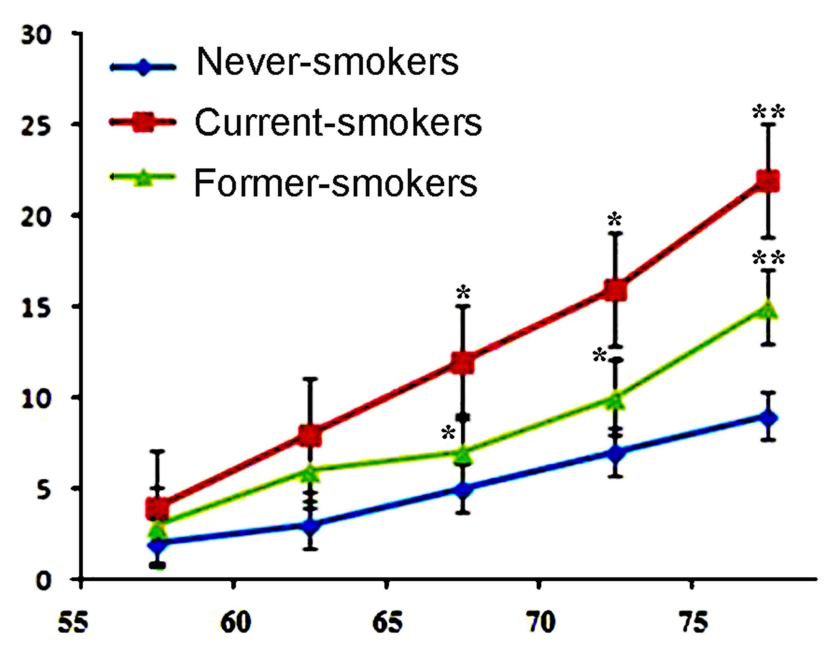

Figure 3 Analysis of plaque scores and age index, which were verified by ultrasonography index. $\mathrm{X}$ axis, age index; $\mathrm{Y}$ axis, scores for calcification and other plaque morphology. $* \mathrm{P}<0.05, * * \mathrm{P}<0.01$.

studies. $^{24}$ In addition, the high-frequency ultrasound can clearly distinguish the details of calcification and was used to observe the location and type of calcification, suggesting combination with two diagnosis tools could offer better understanding of plaque calcification for current smoking patients. Moreover, under some urgent conditions, high-frequency ultrasound itself could preliminarily provide plaque calcification information, and compared with CT scanning, high-frequency ultrasound could be more portable and feasible in emergency treatment with clinical significance.

Previous study has found that the average values of the total calcification of active smokers and passive smokers with essential hypertension were significantly higher than that of the patients without exposure to cigarette smoke. $^{25,26}$ In another study using high-resolution magnetic resonance imaging to evaluate the carotid atherosclerosis plaque distribution, total plaque length was significantly positively associated with the history of smoking. ${ }^{27}$ It has been considered that the process of atherosclerotic plaque calcification presents a number of similarities with the physiological processes that occur in bone, involving both osteoblasts and osteoclasts-like arterial cells. ${ }^{28}$ Moreover, it was reported that risk factors, such as hypertension, cigarette smoke and diabetes, can cause the destabilization of the atheromatic plaque acting on calcification process as well as inflammation. ${ }^{28}$

We also demonstrated that, compared with former smokers, current smokers had higher incidence of abnormal surface morphology, which included irregular surface. Similar with other studies, irregular plaque still induced higher vascular injury incidence even in the smoking history patients. In the literature, the effect of irregular plaque on plaque calcification has not been clarified yet. More specifically, plaque calcification was not explored on the relationship to irregular plaque in current smoking. Our analysis showed that, with higher ultrasonography-determined irregular plaque, the risk of plaque calcification indeed increased dramatically.

\section{Limitations}

Our study has some limitations. This study was conducted on a retrospective basis and represented a single center experience. Despite having found some phenomenon and association, we could not draw a causal relationship between current smoking and development of plaque calcification. The effect antihypertensive, lipid lowering and hypoglycemic drugs, which might affect our results were not evaluated in this study. Accordingly, further randomized clinical trial is needed. In addition, establishment of animal model is essential for better understanding of detailed mechanisms.

\section{Conclusion}

Current smoking was positively associated with plaque calcification onsets, and smoking cessation could efficiently

Table 3 Related Risk Factors Between Calcification and Plaque Surface Morphology

\begin{tabular}{|l|l|l|l|l|l|l|}
\hline \multirow{2}{*}{} & \multicolumn{2}{l|}{ Presence of Calcification } & \multicolumn{2}{l|}{ Surface Morphology } \\
\cline { 2 - 7 } & P value & OR & Cl 95\% & P value & OR & CI 95\% \\
\hline Age & 0.08 & 1.04 & $0.99-1.09$ & 0.02 & 1.04 & $1.0-1.07$ \\
BMI & 0.28 & 0.93 & $0.82-1.06$ & 0.14 & 1.08 & $0.98-1.18$ \\
Hypertension & 0.43 & 0.07 & $0.29-1.69$ & 0.73 & 1.11 & $0.61-2.02$ \\
Hyperlipidemia & 0.76 & 0.89 & $0.40-1.96$ & 0.48 & 1.24 & $0.68-2.25$ \\
Hyperglycemia & 0.13 & 1.91 & $0.83-4.41$ & 0.67 & 0.89 & $0.50-1.56$ \\
Current smoking & 0.02 & 3.74 & $1.2-11.6$ & 0.00 & 2.81 & $1.43-5.53$ \\
Former smoking & 0.01 & 0.336 & $0.14-0.78$ & 0.52 & 0.81 & $0.40-1.57$ \\
\hline
\end{tabular}


attenuate such injury. High-frequency ultrasound can clearly distinguish the details of calcification with promising clinical significance for current smoking patients.

\section{Data Sharing Statement}

The datasets used or analyzed during the current study are available from the corresponding author on reasonable request.

\section{Ethical Approval}

This study is approved by the ethics committee of Xuanwu Hospital, Capital Medical University (No.2014018). Written informed consent was obtained. This study was conducted in accordance with the Declaration of Helsinki.

\section{Consent for Publication}

Informed consent was obtained from all individual participants included in the study.

\section{Disclosure}

The authors report no conflicts of interest in this work.

\section{References}

1. Keeley EC, Boura JA, Grines CL. Primary angioplasty versus intravenous thrombolytic therapy for acute myocardial infarction: a quantitative review of 23 randomised trials. Lancet. 2003;361:13-20. doi:10.1016/S0140-6736(03)12113-7

2. Dirksen MT. Drug-eluting vs bare-metal stents in primary angioplasty. Arch Intern Med. 2012;172:611. doi:10.1001/ archinternmed.2012.758

3. Jukema JW, Verschuren JJW, Ahmed TAN, Quax PHA. Restenosis after PCI. Part 1: pathophysiology and risk factors. Nat Rev Cardiol. 2011;9:53-62. doi:10.1038/nrcardio.2011.132

4. Turak O, Ozcan FF, Isleyen A, et al. Usefulness of the neutrophil-tolymphocyte ratio to predict bare-metal stent restenosis. Am J Cardiol. 2012;110:1405-1410. doi:10.1016/j.amjcard.2012.07.003

5. Li -J-J, Ren Y, Chen K-J, et al. Impact of C-reactive protein on in-stent restenosis: a meta-analysis. Tex Heart Inst J. 2010;37:49-57.

6. Bolca O, Goung B, Ozcan KS, et al. The neutrophil-tolymphocyte ratio is associated with bare-metal stent restenosis in STEMI patients treated with primary PCI. Coron Artery Dis. 2015;26:402-408. doi:10.1097/MCA.0000000000000254

7. Alfaleh HF, Alsuwaida AO, Ullah A, et al. The prognostic impact of in-hospital worsening of renal function in patients with acute coronary syndrome. Int $J$ Cardiol. 2013;167:866-870. doi:10.1016/j. ijcard.2012.01.097

8. Amin AP, Spertus JA, Reid KJ, et al. The prognostic importance of worsening renal function during an acute myocardial infarction on long-term mortality. Am Heart J Mosby. 2010;160:1065-1071. doi:10.1016/j.ahj.2010.08.007

9. Goldberg A, Hammerman H, Petcherski S, et al. Inhospital and 1-year mortality of patients who develop worsening renal function following acute ST elevation myocardial infarction. Am Heart $J$. 2005;150:330-337. doi:10.1016/j.ahj.2004.09.055

10. Stefanini GG, Taniwaki M, Kalesan B, et al. The impact of renal impairment on long-term safety and effectiveness of drug-eluting stents. PLoS One. 2014;9:1-9. doi:10.1371/journal.pone.0106450
11. Cassese S, Byrne RA, Tada T, et al. Incidence and predictors of restenosis after coronary stenting in 10004 patients with surveillance angiography. Heart. 2014;100:153-159. doi:10.1136/heartjnl-2013304933

12. Best PJM, Berger PB, Davis BR, et al.; PRESTO Investigators. Impact of mild or moderate chronic kidney disease on the frequency of restenosis: results from the PRESTO trial. $J$ Am Coll Cardiol. 2004;44:1786-1791. doi:10.1016/j.jacc.2004.07.052

13. Flenaugh EL. Tobacco smoking in China: a pulmonary health crisis. Curr Opin Pulm Med. 2019;25(2):188-191. doi:10.1097/ MCP. 0000000000000556

14. Marenzi G, Lauri G, Assanelli E, et al. Contrast-induced nephropathy in patients undergoing primary angioplasty for acute myocardial infarction. J Am Coll Cardiol. 2004;44:1780-1785. doi:10.1016/j. jacc.2004.07.043

15. Hao Y, Liu J, Liu J, et al., CCC-ACS Investigators. Rationale and design of the Improving Care for Cardiovascular Disease in China (CCC) project: a national effort to prompt quality enhancement for acute coronary syndrome. BMJ Open. 2018;8(7):e020968. doi:10.1136/bmjopen-2017-020968

16. Rubartelli P, Niccoli L, Verna E, et al. Stent implantation versus balloon angioplasty in chronic coronary occlusions: results from the GISSOC trial. Gruppo Italiano di Studio sullo Stent nelle Occlusioni Coronariche. J Am Coll Cardiol. 1998;32:90-96. doi:10.1016/S07351097(98)00193-4

17. Seyfarth M, Kastrati A, Mann JFE, et al. Prognostic value of kidney function in patients with ST-elevation and non-ST-elevation acute myocardial infarction treated with percutaneous coronary intervention. Am J Kidney Dis. 2009;54:830-839. doi:10.1053/j. ajkd.2009.04.031

18. Yamaguchi J, Kasanuki H, Ishii Y, et al.; HIJC Investigators. Serum creatinine on admission predicts long-term mortality in acute myocardial infarction patients undergoing successful primary angioplasty: data from the Heart Institute of Japan Acute Myocardial Infarction (HIJAMI) Registry. Circ J. 2007;71:1354-1359. doi:10.1253/ circj. 71.1354

19. Halkin A, Mehran R, Casey CW, et al. Impact of moderate renal insufficiency on restenosis and adverse clinical events after paclitaxel-eluting and bare metal stent implantation: results from the TAXUS-IV trial. Am Heart J. 2005;150:1163-1170. doi:10.1016/j.ahj.2005.01.032

20. Wang CH, Zhang SY, Fang Q, et al. Renal dysfunction and hsCRP predict long-term outcomes of percutaneous coronary intervention in acute myocardial infarction. Am J Med Sci. 2015;349:413-420. doi:10.1097/MAJ.0000000000000430

21. Zhang RY, Zhu ZB, Zhang Q, et al. Impact of moderate or severe renal insufficiency on long-term outcomes in patients undergoing drug-eluting stent based coronary intervention. Int $J$ Cardiol. 2009;136:72-79. doi:10.1016/j.ijcard.2008.04.028

22. Garg P, Charytan DM, Novack L, et al. Impact of moderate renal insufficiency on restenosis and adverse clinical events after sirolimus-eluting and bare metal stent implantation (from the SIRIUS trials). Am J Cardiol. 2010;106:1436-1442. doi:10.1016/j. amjcard.2010.07.011

23. Sahin I, Gungor B, Can MM, et al. Lower levels of Vitamin D is correlated with higher incidence of contrast-induced nephropathy (CIN) in patients undergoing coronary angiography. Can J Cardiol. 2014;30:428-433. doi:10.1016/j.cjca.2013.12.029

24. Sahin I, Karabulut A, Avci II, et al. Contribution of platelets indices in the development of contrast-induced nephropathy. Blood Coagul Fibrinol. 2014;26(October2009):1-4.

25. Gać P, Jaźwiec P, Mazur G, Poręba R. Exposure to cigarette smoke and the carotid arteries calcification index in patients with essential hypertension. Cardiovasc Toxicol. 2017;17(3):335-343. doi:10.1007/ s12012-016-9391-x 
26. Gać P, Jaźwiec P, Mazur G, Poręba R. Exposure to cigarette smoke and the morphology of atherosclerotic plaques in the extracranial arteries assessed by computed tomography angiography in patients with essential hypertension. Cardiovasc Toxicol. 2017;17(1):67-78. doi:10.1007/s12012-015-9357-4

27. Murata K, Murata N, Chu B, et al., CARE-II Study Collaborators. Characterization of carotid atherosclerotic plaques using 3-dimensional MERGE magnetic resonance imaging and correlation with stroke risk factors. Stroke. 2020;51(2):475-480. doi:10.1161/ STROKEAHA.119.027779
28. Scimeca M, Anemona L, Granaglia A, et al. Plaque calcification is driven by different mechanisms of mineralization associated with specific cardiovascular risk factors. Nutr Metab Cardiovasc Dis. 2019;29(12):1330-1336.

\section{Publish your work in this journal}

The International Journal of General Medicine is an international, peer-reviewed open-access journal that focuses on general and internal medicine, pathogenesis, epidemiology, diagnosis, monitoring and treatment protocols. The journal is characterized by the rapid reporting of reviews, original research and clinical studies across all disease areas. The manuscript management system is completely online and includes a very quick and fair peer-review system, which is all easy to use. Visit http://www.dovepress.com/ testimonials.php to read real quotes from published authors.

Submit your manuscript here: https://www.dovepress.com/international-journal-of-general-medicine-journal 